\title{
Impact pressures of turbulent high-velocity jets plunging in pools with flat bottom
}

\author{
P. A. Manso - E. F. R. Bollaert · A. J. Schleiss
}

Received: 22 August 2006/Revised: 25 September 2006/ Accepted: 26 September 2006/Published online: 8 November 2006

(C) Springer-Verlag 2006

\begin{abstract}
Dynamic pressures created by the impact of high-velocity turbulent jets plunging in a water pool with flat bottom were investigated. Pressure fluctuations were sampled at $1 \mathrm{kHz}$ at the jet outlet and at the pool bottom using piezo-resistive pressure transducers, jet velocities of up to $30 \mathrm{~m} / \mathrm{s}$ and pool depth to jet diameter ratios from 2.8 to 11.4. The high-velocity jets entrain air in the pool in conditions similar to prototype applications at water release structures of dams. The intermittent character of plunge pool flows was investigated for shallow and deep pools, based on high order moments and time correlations. Maximum intermittency was observed for pool depths at 5.6 jet diameters, which approximate the core development length. Wall pressure skewness was shown to allow identifying the zone of influence of downward and upward moving currents.
\end{abstract}

P. A. Manso - E. F. R. Bollaert · A. J. Schleiss

Laboratory of Hydraulic Constructions,

Ecole Polytechnique Fédérale de Lausanne (EPFL),

1015 Lausanne, Switzerland

E. F. R. Bollaert

e-mail: erik.bollaert@epfl.ch

A. J. Schleiss

e-mail: anton.schleiss@epfl.ch

P. A. Manso ( $\square)$

Instituto Superior Técnico (IST),

Technical University of Lisbon,

DECivil-SHRHA, Gab. 3.43,

Av. Rovisco Pais, 1049-001 Lisbon, Portugal

e-mail: pmanso@civil.ist.utl.pt

\section{List of symbols}

$C_{\mathrm{s}} \quad$ skewness parameter

$D \quad$ jet nozzle diameter

$\mathrm{Fr} \quad$ Froude number $\left[=V_{0} /\left(g D_{0}\right)^{0.5}\right]$

$K \quad$ kurtosis parameter

$L \quad$ jet travel distance in the air

$L_{\mathrm{b}} \quad$ jet break-up length in the air

$p, p^{\prime} \quad$ pressure, pressure fluctuations

$Q \quad$ discharge

$R e \quad$ Reynolds number $\left(=V_{0} D_{0} / v\right)$

$R$ space-time unidirectional correlation function

$\mathrm{Tu} \quad$ turbulence intensity $\left(=u^{\prime} / V\right)$

$V, V_{0}$ average velocity; at nozzle exit

$x \quad$ pool depth measured from surface

$x_{\mathrm{c}} \quad$ core development length

$Y \quad$ pool depth

$y \quad$ radial (horizontal) coordinate

$z, z^{\prime} \quad$ normalized variable of Gaussian pdf $\left(=\frac{p-\mu}{\sigma}\right)$

$\alpha_{1} \quad$ free diffusion core contraction angle

$\alpha_{2} \quad$ free diffusion spreading angle

$v \quad$ kinematic viscosity

$\mu \quad$ mean value of data sample

$\sigma \quad$ standard deviation (or RMS) of data sample

$\rho$ density, discrete normalized autocorrelation

$\Delta \quad$ difference to ensemble statistics

\section{Introduction}

The investigation of impact pressures generated by the diffusion of turbulent high-velocity aerated water jets presents many difficulties for experimental research due to the complex two-phase environment. The 
hydrodynamic flow field is often extrapolated from non-aerated, low turbulence or free diffusion conditions, which are easier to reproduce in laboratory. In dam engineering and water treatment industries, these simplifications may lead to severe miscalculation of dissipative processes and of the dynamic loads acting on the solid boundaries. According to Albertson et al. (1948), jet diffusion is divided in two flow regions: the flow development region $\left(x \leq x_{\mathrm{c}}\right)$, where the jet potential core persists; and the established flow region further downstream $\left(x>x_{\mathrm{c}}\right)$. The diffusion of a plunging jet depends on its entry velocity in the pool, density and turbulence intensity, as well as jet and pool dimensions. These characteristics define the rate of mixing with the surrounding fluid and the distance influenced by the jet, as indicated in Fig. 1. Therefore, the dynamic loads on the pool bottom are a function of jet turbulent characteristics before and at pool entry, the amount of air being entrained, and the pool depth (Ervine et al. 1997). Tests in reduced-scale physical models have shown that increasing pool depth reduces mean pressures at the pool bottom (May and Willoughby 1991; Puertas-Agudo 1994; Melo 2001). However, they do not reliably account for pool aeration due to non-compliance with the Weber similarity criteria. The enhancement in turbulent mixing and in energy dissipation due to air bubble buoyancy is overlooked. To bridge this gap, Ervine et al. (1997) and Bollaert and Schleiss (2003) started investigating plunging jets in facilities producing jet velocities as high as those encountered in engineering applications.

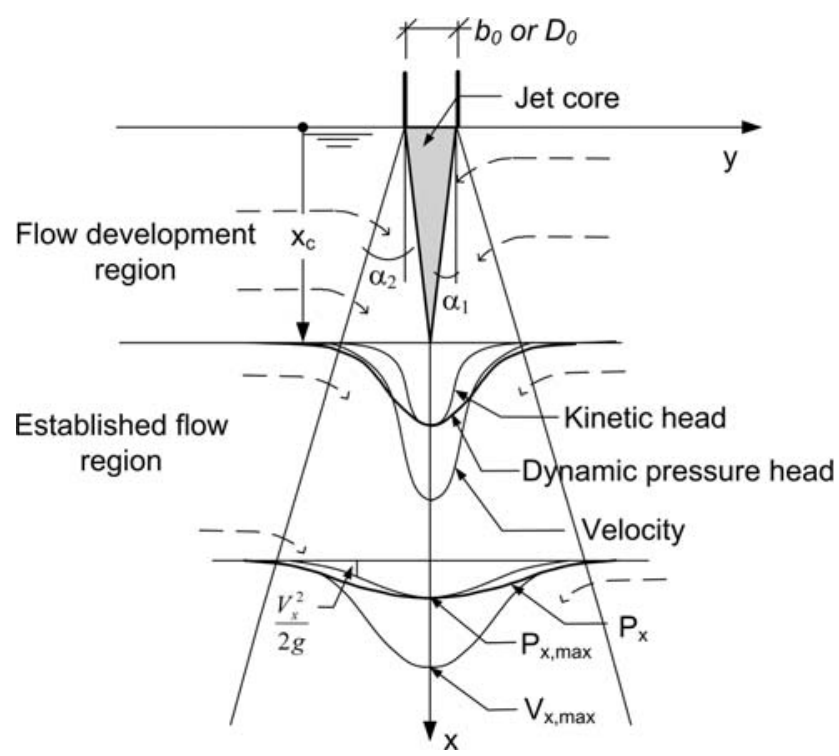

Fig. 1 Free jet diffusion (based on Hartung and Häusler 1973). $\alpha_{1}$ is the core contraction angle, $\alpha_{2}$ is the shear layer boundary spreading angle, and $x_{\mathrm{c}}$ is the core length
Their facilities generate highly aerated and highvelocity pool flows. Due to the difficulties in performing measurements inside the pool under these conditions, research in this area has concentrated on the resulting outcome of the diffusion process, close to the boundaries, i.e., the impact pressures at the pool bottom. Ervine et al. (1997) presented impact pressures statistics using jet velocities of up to $29 \mathrm{~m} / \mathrm{s}$, varying travel distances, initial jet turbulence, jet velocity and pool depths. Bollaert and Schleiss (2003) compared wall pressure measurements at the pool bottom and their propagation inside rock fissures.

In parallel with research in impact pressures, jet air entrainment and jet diffusion in the pool have been investigated, but limited to the developing flow region and low-turbulence jets. Fundamental studies with plunging jets by, among others, McKeogh and Ervine (1981); Ervine and Falvey (1987), present experimental evidence of air concentration decay with pool depth with different break-up degrees at jet entry, but only in free diffusion conditions, i.e., away from the pool bottom. Chanson et al. (2004) studied air-water flow characteristics close to jet entry in pools for undeveloped jets, but with low turbulence intensities (less than $1 \%)$. The influence of jet deflection at the pool bottom and the behaviour of air bubbles when approaching the bottom requires further research. McKeogh and Elsawy (1980) described how bubble penetration is modified due to the presence of an obstacle. However, they did not measure impact pressures. Melo (2002) documented the impact conditions of submerged water jets with artificial air entrainment in lined pools for velocities of up to $10 \mathrm{~m} / \mathrm{s}$, by directly and fully controlling the amount of air being entrained. He confirmed experimentally that air entrainment reduces mean impact pressures. Inversely, pressure fluctuations are enhanced with increasing air entrainment. However, these experiments do not take into consideration the different degrees of jet break-up at pool entry, i.e., the ratio between the travel distance and the core development length in air (Ervine et al. 1997). Jet development in the air depends on the jet characteristics at issuance, namely cross-sectional shape, velocity profile and turbulence intensity. Overall, several studies have approached different relevant topics for two-phase jet diffusion in plunge pools but a comprehensive analysis of dynamic pressures in limited-depth pools combining high velocities, pool aeration and prototype turbulence levels, by avoiding scale effects, is still missing.

This paper presents experimental work with turbulent high-velocity jets plunging in a water pool based on recent work by Manso (2006). High-frequency 
pressure measurements were performed at the jet outlet (i.e., at issuance) and compared with measurements at the pool bottom. The impact pressures generated by rough turbulent jets are described statistically for variable jet velocities and pool depths. Air entrainment and impact pressures are investigated under conditions similar to those found in water releasing structures of large dams. Under these conditions, probability density functions and high-order statistical moments provide unprecedented insight on the intermittent character of pool turbulence.

\section{Experimental work}

\subsection{Pressure measurements at issuance and pool bottom}

To measure dynamic pressures generated by highvelocity jets, an experimental facility, shown in Fig. 2 with a circular basin, was used as described by Bollaert and Schleiss (2003). The jet outlet is circular, with a nozzle exit diameter $D=0.072 \mathrm{~m}$. The pool depth $Y$ is variable according to the height of lateral weirs. The jet diameter is within a $1 / 1$ to $1 / 20$ geometric scale of target prototype applications. The tests conditions are presented in Tables 1 and 2.

The experimental facility is identical to that used by Bollaert and Schleiss (2003) except for a few modifications in the supply system. The placement of a honeycomb grid immediately upstream of the last bend reduced swirling and improved the homogeneity of the flow over the section. An air vent was provided at the highest point of the supply system to prevent the formation of air bubbles during filling of the conduit and to assure a stable operation of the jet. These features improved jet stability, eliminating swirl; the velocity profile at the nozzle exit was almost uniform for velocities from 10 to $30 \mathrm{~m} / \mathrm{s}$ (Manso 2006). Dynamic pressures were measured at the jet nozzle outlet and at the pool bottom. For the former, the pressure transducer was placed in a mobile metallic structure inside the jet right below the nozzle, supported by a rectangular steel frame (Fig. 3).

\subsection{Instrumentation}

Pressure measurements at the jet outlet were made with a piezo-resistive micro-transducer of type Kulite XTL-190-17BAR-A and a sampling frequency of $2 \mathrm{kHz}$ for $32.5 \mathrm{~s}$. The transducer has a $3 \mathrm{~mm}$ diameter diaphragm and an accuracy of $0.1 \%$ of the full-scale output (17 bar absolute) due to non-linearity and hysteresis (i.e., max. 0.017 bar $\approx 0.17 \mathrm{~m}$ ). Discharge measurements were performed with an electromagnetic flowmeter of $1 \%$ accuracy. Pressure transducers of type Kulite XTM-190-17BAR-A were flush mounted on the pool bottom. The pressure signal was sampled at $1 \mathrm{kHz}$ during $65 \mathrm{~s}$ (each run). Readings have a potential combined non-linearity, hysteresis and repeatability $(\mathrm{CNL} \& \mathrm{H})$ of $1 \%$ of the full-scale output $(\mathrm{FSO}=17$ bar A $)$. A combined error analysis showed mean pressures may vary within maximum $80 \%$ of the incoming jet kinetic energy for $V=9.8 \mathrm{~m} / \mathrm{s}$ and $9 \%$ for $30 \mathrm{~m} / \mathrm{s}$, respectively. These envelope error margins include variations of pool surface, atmospheric pressure, transducer's zero drift, non-linearity and hysteresis and calibration. They decrease with increasing jet velocity.

\subsection{Ergodic pressure measurements}

An ergodicity analysis was carried out to determine the minimum total data acquisition time required to obtain reliable estimates of the first two moments of a data
Fig. 2 Schematic plot of the experimental facility. 1 Jet outlet, 2 basin, 3 and 4 outflow; 5 digital acquisition (DAQ) system for pressure transducers, placed every $25 \mathrm{~mm}$, starting at $y / D=0.35$, and 6 air vent and honeycomb grid

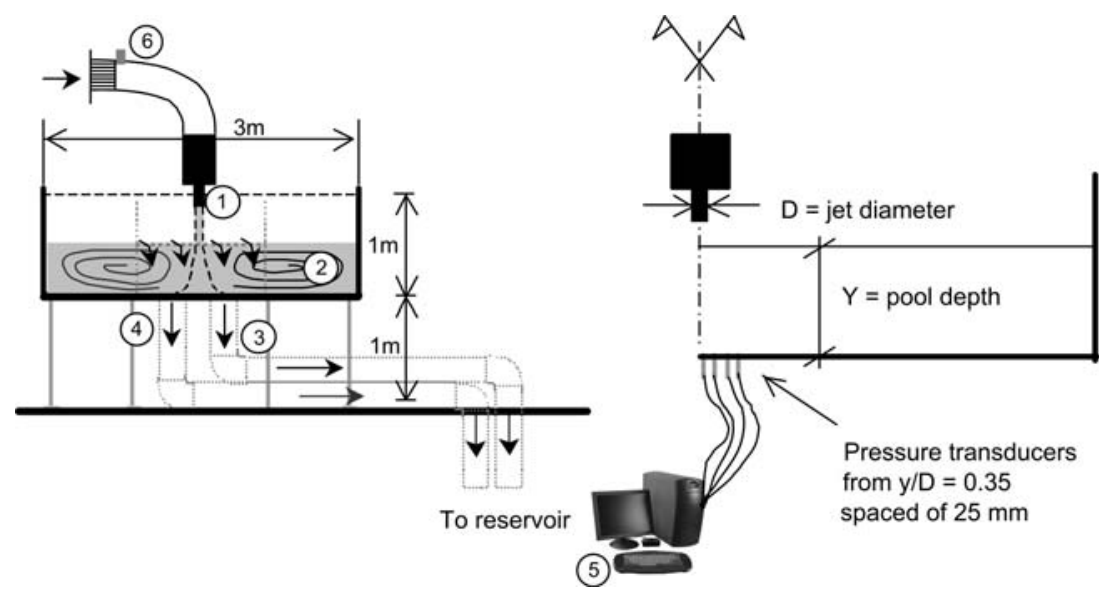


Table 1 Test conditions, where $Q$ is discharge, $V_{0}$ mean exit velocity, $R e$ Reynolds number and $F r$ Froude number

\begin{tabular}{lcccc}
\hline Test & $Q(1 / \mathrm{s})$ & $V_{0}(\mathrm{~m} / \mathrm{s})$ & $\operatorname{Re} \times 10^{5}(-)$ & $\operatorname{Fr}(-)$ \\
\hline Flat bottom & 30 & 7.4 & 4.6 & 8.8 \\
& 40 & 9.8 & 6.2 & 11.7 \\
& 50 & 12.3 & 7.7 & 14.6 \\
& 60 & 14.7 & 9.2 & 17.5 \\
& 70 & 17.2 & 10.8 & 20.5 \\
& 80 & 19.6 & 12.3 & 23.4 \\
& 90 & 22.1 & 13.8 & 26.3 \\
& 100 & 24.6 & 15.4 & 29.2 \\
& 110 & 27.0 & 16.9 & 32.1 \\
& 120 & 29.5 & 18.5 & 35.1 \\
\hline
\end{tabular}

Table 2 Pool water level $Y$ and jet fall $L$ conditions for the tested velocities; $Y / D$ is the relative pool depth and $L / L_{\mathrm{b}}$ the relative degree of jet break-up

\begin{tabular}{llll}
\hline$Y(\mathrm{~m})$ & $Y / D(-)$ & $L(\mathrm{~m})$ & $L / L_{\mathrm{b}}(-)$ \\
\hline 0.075 & 1.0 & 0.625 & $0.40-0.44$ \\
0.20 & 2.8 & 0.50 & $0.32-0.35$ \\
0.30 & 4.2 & 0.40 & $0.26-0.28$ \\
0.40 & 5.6 & 0.30 & $0.19-0.21$ \\
0.50 & 6.9 & 0.20 & $0.13-0.14$ \\
0.60 & 8.3 & 0.10 & $0.06-0.07$ \\
0.67 & 9.3 & 0.03 & 0.02 \\
0.82 & 11.4 & Submerged & - \\
0.87 & 12.1 & Submerged & - \\
\hline
\end{tabular}

series, i.e., the mean $\mu$ and the standard deviation $\sigma$, within reasonable variation limits of the corresponding ensemble moments. An evaluation of the range of variation of the skewness and kurtosis was also performed. The skewness and kurtosis reflect the importance of extreme pressure values in the probabilistic pressure distribution, defined as

$$
\begin{aligned}
C_{\mathrm{s}} & =\frac{\sum_{i=1}^{N}\left(p_{i}-\bar{p}\right)^{3}}{(N-1) \operatorname{RMS}\left(p^{\prime}\right)^{3}}, \\
K & =\frac{\sum_{i=1}^{N}\left(p_{i}-\bar{p}\right)^{4}}{(N-1) \operatorname{RMS}\left(p^{\prime}\right)^{4}}-3 .
\end{aligned}
$$

Obtaining estimates of the latter moments within the same limits as for $\mu$ and $\sigma$ required longer-duration runs and was not the main objective of the study. The sampling frequency was $1 \mathrm{kHz}$, due to the significant spectral energy content at frequencies as high as $300 \mathrm{~Hz}$. Successive runs were performed up to a net maximum duration of about $0.5 \mathrm{~h}$. This analysis considered instantaneous pressures at the pool bottom $(y / D=0.35$ and 0.69$)$ for different pool depths and jet velocities. The mean value, the standard deviation, the skewness $C_{\mathrm{s}}$ and the kurtosis $K$ parameters of each single run, as well as the cumulated statistics for the sum of $n$ runs, were computed for each transducer. The entire set of data was divided in sub-sets of given duration, e.g. equal to the sum of $2,3,5$, and 20 consecutive runs, comparing the statistics of such subsets with the ensemble statistics obtained from entire dataset. The larger the duration of the sub-sets, the lower the range of variation (Fig. 4). This analysis allowed selecting a cumulative acquisition time of about $3 \mathrm{~min}$ at $1 \mathrm{kHz}$; this total data acquisition time was then used systematically during the test campaign throughout the experiments. A selection of test cases is presented in Table 3.
Fig. 3 Experimental set-up used for pressure measurements at the jet issuance section. a Schematic view, $\mathbf{b}$ photo of the measuring frame under the jet nozzle, $\mathbf{c}$ honeycomb grid placed 25 nozzle diameters upstream, and $\mathbf{d}$ measuring points across jet diameter spaced of 4-5 $\mathrm{mm}$ each
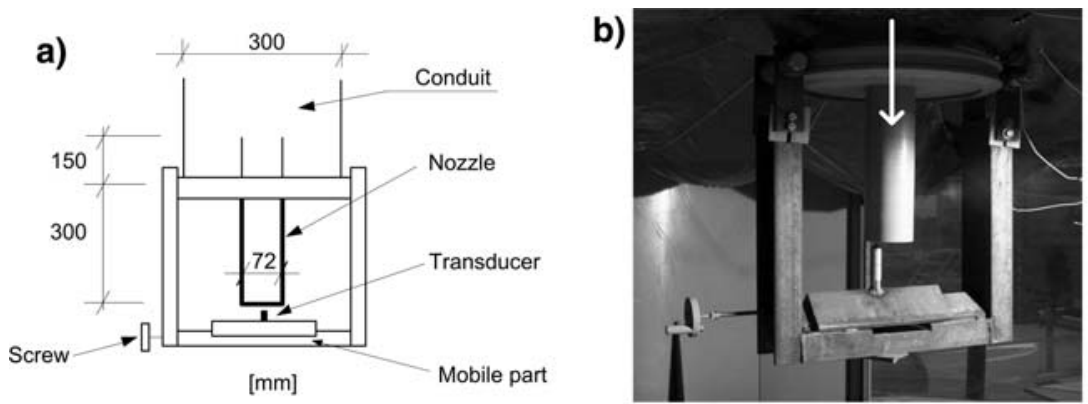

c)

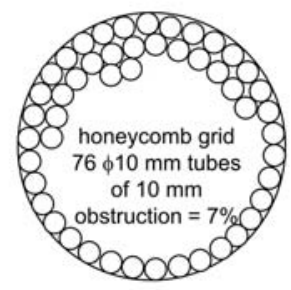

d)

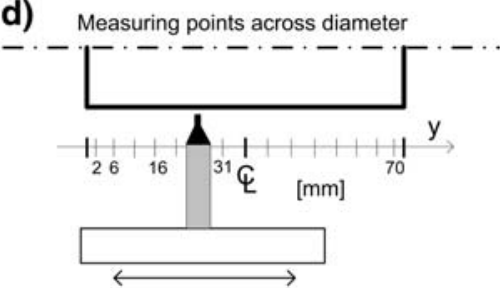



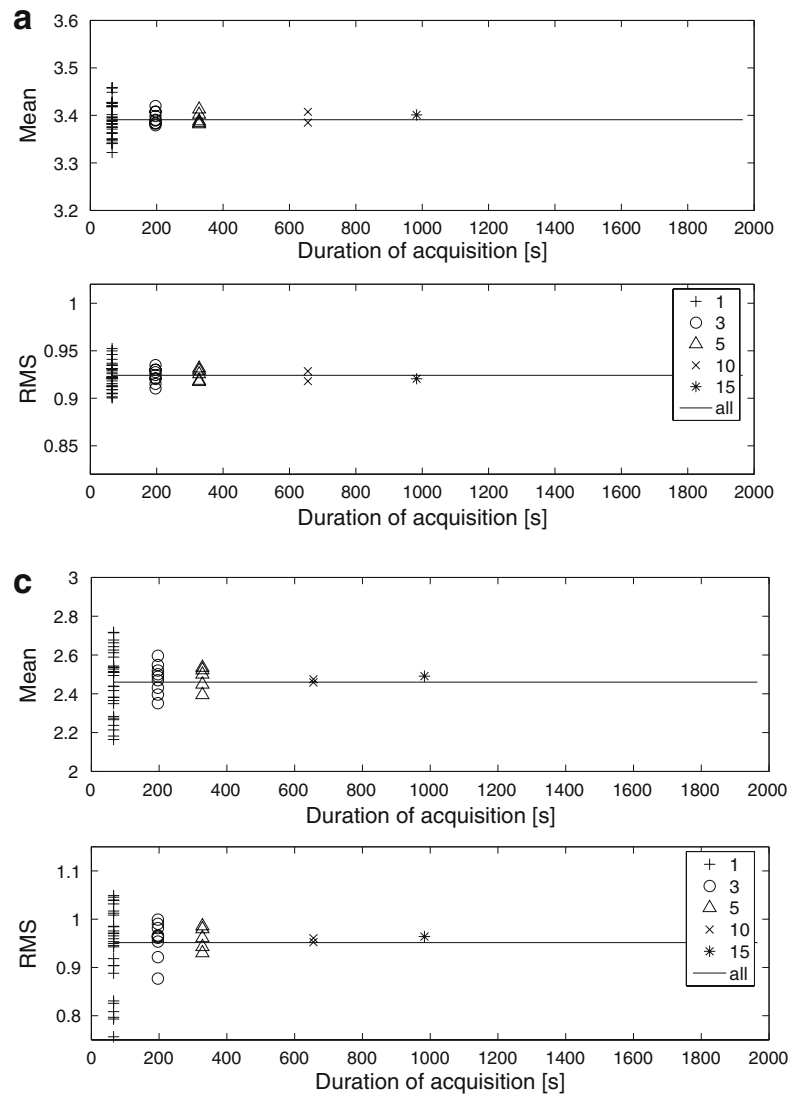

Fig. 4 Estimates of the mean (in bar), standard deviation (in bar $^{2}$ ), skewness and kurtosis of pressure data for different duration of the sub-sets (i.e. $1,3,5,10$, and 15 runs) at $y / D=0.35$
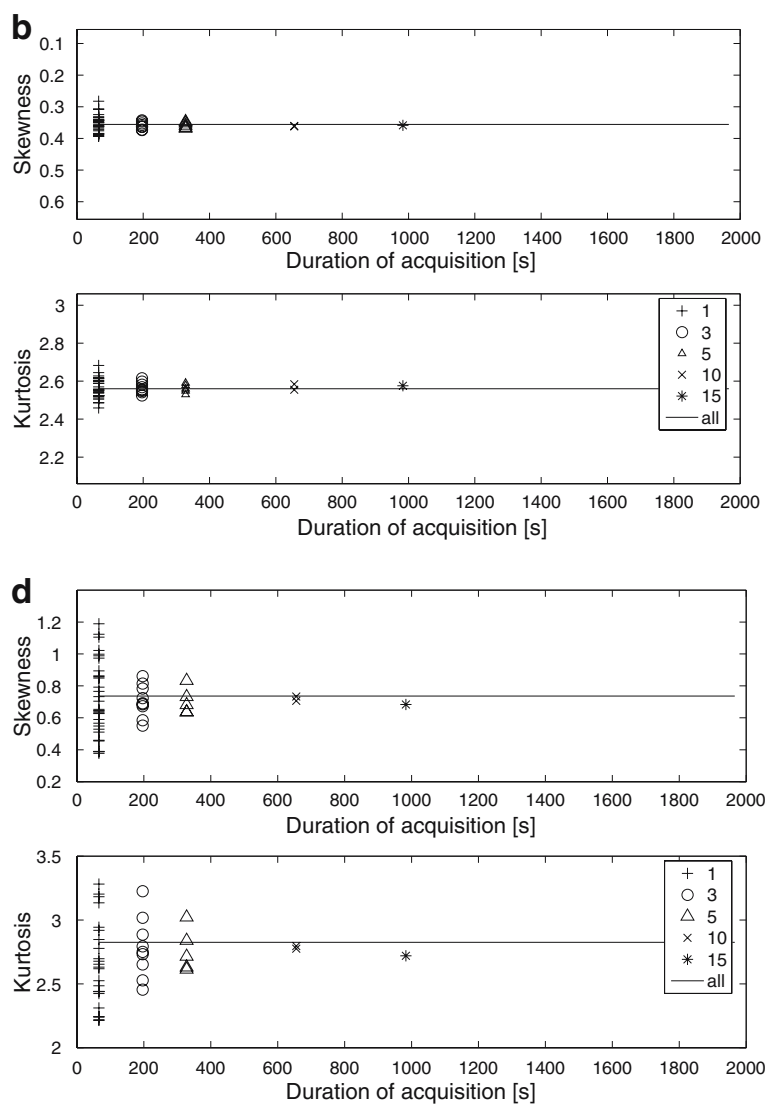

compared with ensemble statistics (straight line). Rows are: top, for shallow pool $(Y / D=5.6)$; bottom, for deep pool $(Y / D=9.3)$

Table 3 Selection of long duration test for ergodicity analysis ( $n=$ number of runs)

\begin{tabular}{llllllllll}
\hline Test & $y / D$ & $Y / D$ & $V(\mathrm{~m} / \mathrm{s})$ & $n$ & Samples & $\Delta \mu$ & $\Delta \sigma$ & $\Delta C_{\mathrm{s}}$ & $\Delta K$ \\
\hline Shallow pool & 0.69 & 5.6 & 27 & 30 & 65,536 & $<1 \%$ & $<2 \%$ & $<10 \%$ \\
Deep pool & 0.69 & 9.3 & 27 & 30 & 65,536 & $<6 \%$ & $<10 \%$ & $<32 \%$ & $<2 \%$ \\
\hline
\end{tabular}

The number of samples varies between 32,768 and 65,536 . Comparison between moments after 3 min of cumulated acquisition time and ensemble moments obtained from cumulated $30 \mathrm{~min}$

\section{Impact pressures}

Impact conditions reflect the degree of jet development in the pool. For shallow pools, the jet potential core impacts the pool bottom below the jet. In deep pools, a developed jet impacts the bottom. The transition from one case to the other occurs at depths $x$ of 4-6 times the jet diameter at entry in the pool (Ervine et al. 1997). This transition depends on the mixing rate between the jet and the pool, which depends on the degree of jet turbulence, on jet velocity and on the density gradient between the jet and the pool. The corresponding flow patterns in limited-depth pools with flat bottom are quite different. For shallow pools, the wall jets created by plunging jet deflection at impact have velocities close to those of the jet. In very shallow pools (e.g. $Y / D=1.0$ ), the pool water was spread open originating a circular hydraulic jump. As the pool depth increased, the jump's front came closer to the jet eventually originating a submerged hydraulic jump. In deep pools, a turbulent shear layer hits the pool bottom, being surrounded by large recirculating eddies; the pool surface is less disturbed.

A selection of tests was used to study pressure statistics (mean value, standard deviation, maxima, minima, skewness and kurtosis) for low and high velocities (approx. 10 and $30 \mathrm{~m} / \mathrm{s}$ ), from measurements at the nozzle exit and after diffusion for the shallow 
Table 4 Statistics of selected tests (at issuance, each file has 32,768 points sampled at $0.5 \mathrm{kHz}$ and at impact with the bottom 65,536 points sampled at $1 \mathrm{kHz}$ )

\begin{tabular}{|c|c|c|c|c|c|c|c|c|c|c|c|c|}
\hline $\begin{array}{l}\text { Measurement } \\
\text { position }\end{array}$ & $Y / D$ & $y / D(-)$ & $V_{0}(\mathrm{~m} / \mathrm{s})$ & $\begin{array}{l}P_{\text {mean }} \\
\text { (bar) }\end{array}$ & $\begin{array}{l}\mathrm{RMS}_{p} \\
\text { (bar) }\end{array}$ & $\begin{array}{l}p_{\text {max }}^{\prime} \\
\text { (bar) }\end{array}$ & $\begin{array}{l}p_{\text {min }}^{\prime} \\
\text { (bar) }\end{array}$ & $C_{\mathrm{s}}(-)$ & $K(-)$ & $z_{\text {max }}^{\prime}(-)$ & $z_{\text {min }}^{\prime}(-)$ & $\mathrm{Tu}(-)$ \\
\hline \multirow[t]{2}{*}{ At issuance } & & 0.0 & 11.62 & 1.660 & 0.110 & 0.563 & 1.080 & -0.85 & 3.67 & 5.11 & -9.80 & 0.081 \\
\hline & & & 30.73 & 6.016 & 0.381 & 2.389 & 3.311 & -0.62 & 1.38 & 6.27 & -8.69 & 0.041 \\
\hline \multirow[t]{4}{*}{ Pool bottom } & 2.78 & 0.35 & 9.82 & 1.254 & 0.077 & 0.237 & 0.420 & -0.96 & 1.22 & 3.09 & -5.46 & 0.080 \\
\hline & $(0.20 \mathrm{~m})$ & & 29.47 & 5.274 & 0.737 & 2.208 & 4.171 & -1.13 & 1.57 & 3.00 & -5.66 & 0.085 \\
\hline & 9.31 & 0.35 & 9.82 & 0.988 & 0.073 & 0.415 & 0.195 & 0.91 & 1.09 & 5.68 & -2.67 & \\
\hline & $(0.67 \mathrm{~m})$ & & 29.47 & 3.102 & 1.042 & 4.532 & 2.303 & 0.77 & -0.03 & 4.35 & -2.21 & \\
\hline
\end{tabular}

$C_{\mathrm{s}}$ is the skewness coefficient, $K$ the flatness excess kurtosis, and $z_{\text {min }}^{\prime}$ and $z_{\text {max }}^{\prime}$ are the minimum and maximum values of the Gaussian distribution variable

$(Y / D=2.8)$ and deep $(Y / D=9.3)$ pools. Table 4 shows that pressure distributions deviate from the Gaussian distribution on the tails. Surprisingly, skewness values are negative at the jet axis. For compact jets (assumed as jets with break-up degree of less than $0.50)$, positive skewness would be expected, showing direct impact in the pressure transducer tip. However, jet reflection close to the stagnation point inverts the flow direction and the sign of the skewness parameter accordingly.

\subsection{Turbulence intensity}

The turbulence intensity of the jet at the nozzle exit $\mathrm{Tu}$ ) is a key parameter in the definition of jet behaviour in the air and inside the pool (Ervine and Falvey 1987). It is defined as $\mathrm{Tu}=u^{\prime} / V$, where $u^{\prime}$ is the rootmean-square (RMS) value of the axial velocity fluctuations and $V$ is the section-averaged axial velocity. Arndt and Ippen (1970) proposed computing Tu from pressure fluctuations using:

$\mathrm{Tu}=\frac{\operatorname{RMS}(u \prime)}{V}=\frac{\sqrt{\overline{p^{\prime 2}}}}{\rho V^{2}}=\frac{\operatorname{RMS}\left(p^{\prime}\right)}{\rho V^{2}}$

where the standard deviation of the velocity fluctuations $u^{\prime}$ is obtained from the standard deviation of the pressure fluctuations $p^{\prime}$. Higher order terms are neglected when converting pressures into velocities with an estimated error of less than $5 \%$ for a turbulence intensity level of $10 \%$, which is considered acceptable regarding the expected prototype turbulence intensities. Tu estimates at the pool bottom are obtained from pressure measurements at the stagnation point using the same procedure, assuming the validity of Eq. 3 in shallow pools.

At issuance, $\mathrm{Tu}$ between 4 and $8 \%$ were obtained for velocities ranging from 10 to $30 \mathrm{~m} / \mathrm{s}$-Table 4 and thus the produced jets are considered "rough jets". The lowest $\mathrm{Tu}$ corresponds to the highest velocity. The higher the velocity, the more uniform is the velocity profile (Manso 2006).

For $Y / D=2.8$, the jet core impacts the bottom and $\mathrm{Tu}$ at impact is about $8 \%$. Compared to $\mathrm{Tu}$ at issuance, this is about the same for $V=10 \mathrm{~m} / \mathrm{s}$ but doubled for $30 \mathrm{~m} / \mathrm{s}$. A detailed time series analysis showed evidence of intense pool surface instabilities generated by bottom wall jet reflection at the basin walls. Pool surface instabilities disturbed the jet's entry in the pool and increased $\mathrm{Tu}$ at impact for the highest velocities tested. This is a typical facility artefact. For the range of tested velocities, the degree of break-up of the jet is $0.32-0.35$ at the entrance of the pool and the jet core cuts through the pool. Jet deformation in the air is small, i.e. for these values of $\mathrm{Tu}$, the core at entry in the pool is $\pm 70 \%$ of $D$ and the outer diameter is not more than $60 \%$ larger than $D$ as estimated according to Ervine et al. (1997).

\subsection{Pressure distribution for variable pool depths}

Pressure measurements close to the jet axis $(y / D=0.35)$ are negatively skewed at the nozzle exit and at the pool bottom in shallow pools (e.g. $Y / D=2.8)$, whereas they are positively skewed at the pool bottom in deep pools (e.g. $Y / D=9.3)$. Jet core impact conditions are thus characterized by negative skewness and positive (excess) kurtosis. For larger pool depths, skewness became positive and kurtosis approached zero (Table 4). In Fig. 5, empirical probability density functions (epdf) are compared with the corresponding Normal (Npdf) and Gumbel (Gpdf) fits. The Normal (or Gaussian) distribution is often assumed valid for engineering practice. There is a growing interest to know how accurate this assumption is. The Gumbel pdf is also quite widely used to estimate extreme values and has the practical advantage of depending (as the Normal pdf does) on only two parameters (mean value and standard deviation). It has been previously used for the analysis of inde- 

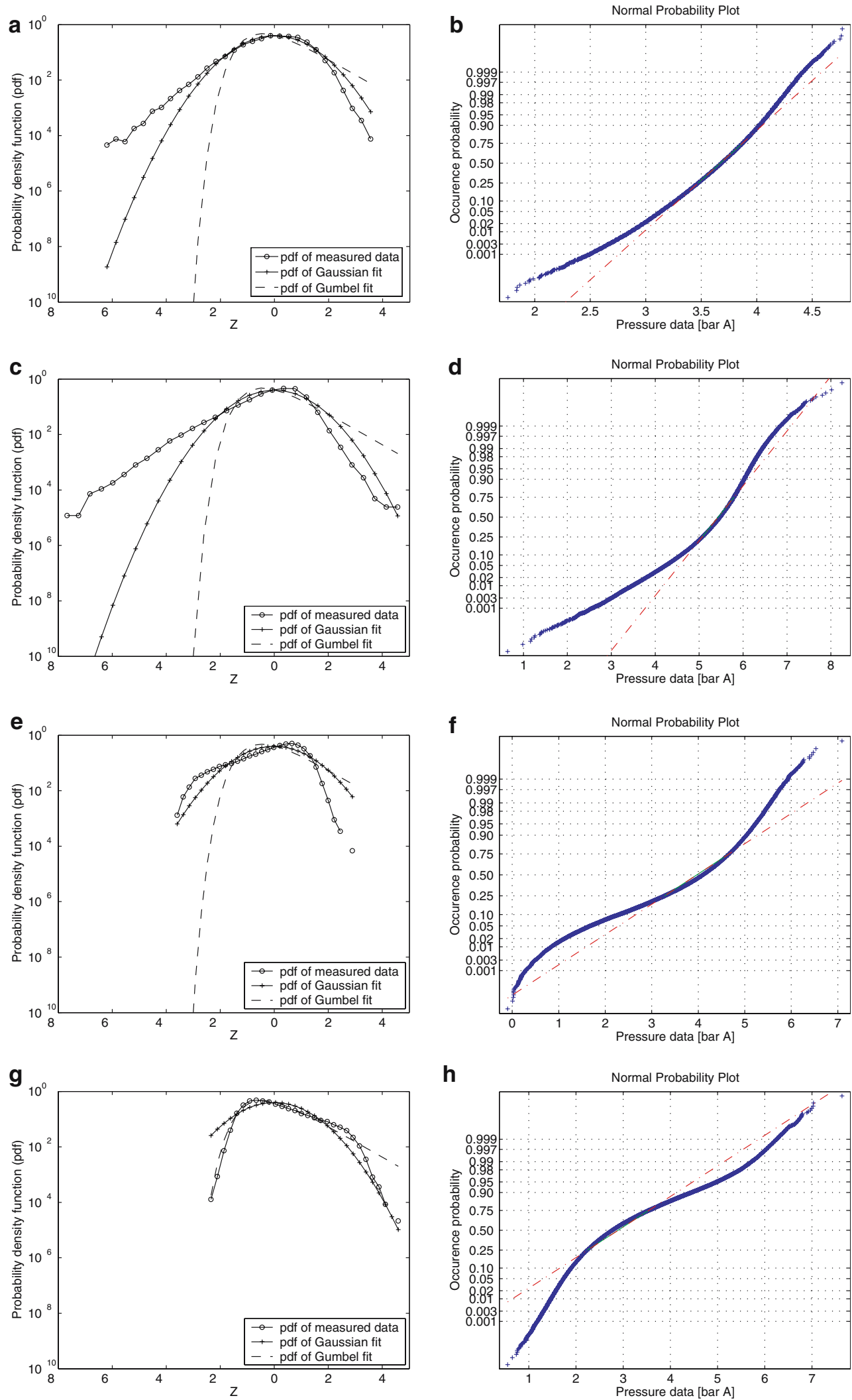

h

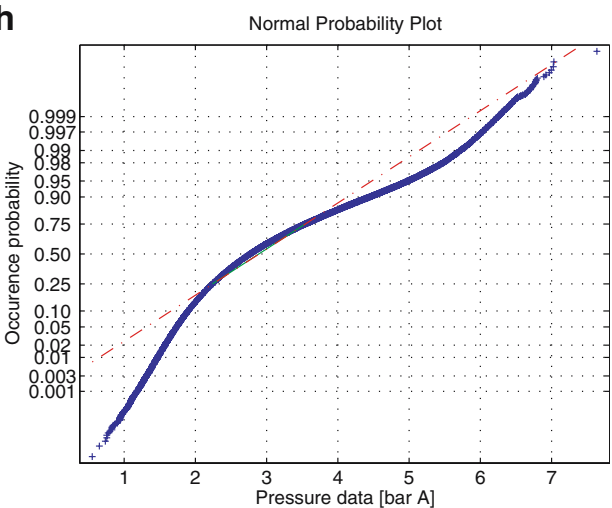

Fig. 5 Left empirical density functions of pressure measurements at the pool bottom $(y / D=0.35)$ compared with the respective normal and gumbel fits. Right direct comparison between empirical density functions and corresponding Normal

fits. Data series: a and b $Y / D=2.8, V=24.6 \mathrm{~m} / \mathrm{s} ; \quad \mathbf{c}$ and d $Y / D=5.6, V=29.5 \mathrm{~m} / \mathrm{s} ;$ e and f $Y / D=6.9, V=29.5 \mathrm{~m} / \mathrm{s}$; g and h $Y / D=9.3, V=29.5 \mathrm{~m} / \mathrm{s}$ 
pendent (i.e., non correlated) extreme pressure values obtained at reduced laboratory scale in 24-hour duration runs (Toso and Bowers 1988). However, due to the small duration of the herein presented nearprototype measurements, their comparison with the Gumbel extreme probability distribution is merely exploratory. Figure 5 shows that the experimental data follow Normal distributions only within $\pm 1.5 \sigma$. The semilog-scale epdfs in the left hand side column show the decrease in importance of the negative extreme values tail for increasing pool depths $(Y / D$ values of 2.8, 5.6, 6.9 and 9.3). The right hand side column shows that a Gaussian fit is acceptable for probabilities of approximately $0.05-0.25$ to $0.75-0.90$, all cases considered (Table 5). The evolution from negative to positive skewness at $y / D=0.35$ with increasing pool depth follows the behaviour of the negative and positive tails of the pdfs. Table 5 presents also the differences in terms of the number of standard deviations between the pressure estimates using a Normal or Gumbel distribution for probabilities of 0.1 and $99.9 \%$. For both the negative and positive tails, the largest difference between estimates from a Gaussian fit and the measured data pdfs were obtained for $Y / D=9.3$.

Figure 6 shows that $C_{\mathrm{s}}$ is negative at stagnation for $Y \leq<8.3 D$. These pools are herein defined as shallow and transitional pools. For deep pools, skewness becomes positive. Therefore, a large submergence is required to allow jet development before impact. For shallow and transitional pools, $C_{\mathrm{s}}$ is positive at $y / D=2.08$, reflecting a more developed turbulent flow pattern (i.e. impact of the turbulent shear layer outside the jet core). It tends to zero for deep pools, indicating that local flow streamlines are quasi-parallel to the bottom. At stagnation, $K$ is positive for shallow and transitional pools, maximum for $Y / D=5.6$ and close to zero for deep pools. This reflects some instability for shallow pools, high intermittent character for $Y / D=5.6$ and the impact of a well-developed shear layer for deep pools. At $y / D=2.08, K$ reflects important surface oscillations in shallow pools (due to the presence of an hydraulic jumps or by wall jet reflection at the side walls), hampered in deep pools.

In terms of skewness, core jet impact conditions (observed at issuance and for $Y / D=2.8$ ) correspond to negative $C_{\mathrm{s}}$ at stagnation, while developed jet impact conditions $(Y / D=9.3)$ correspond to positive $C_{\mathrm{s}}$. Such counter-intuitive results can be explained in terms of the local flow pattern. For that purpose, autocorrelation functions were computed. The space-time unidirectional correlation function is defined as:

$R(x, \Delta x, \tau)=\lim _{T \rightarrow \infty} \int_{0}^{T} p^{\prime}(x, t) \cdot p^{\prime}(x+\Delta x, t+\tau) \mathrm{d} t$,

where $p \prime(\mathrm{x}, \mathrm{t})=p(x, t)-\bar{p}$. The discrete normalized autocorrelation at $y / D=0.35$ was estimated by:

$\rho(x, 0, \tau)=\frac{R(x, 0, \tau)}{\sigma^{2}}$

The initially rapid decay observed in Fig. 7 provides information on small flow structures, whereas the slow decaying tail represents a second (larger) structure. This is in good agreement with the analogous observations of Carreras et al. (1998). For a shallow pool $(Y / D=2.8$ and negative skewness at $y / D=0.35)$, the sharp decay of the autocorrelation structure corresponds to the description of small flow structures resulting from flow deflection close to the stagnation point. In fact, if one assumes an $8^{\circ}$ angle for core contraction inside the pool and follows the plunging jet theory by Ervine and Falvey (1987), the transducer placed at $y / D=0.35$ is influenced by the jet core. This model was, however, established for free diffusion conditions and does not account for jet deflection close to the bottom. In the present case, the transducer readings are influenced by a fluctuating behaviour of

Table 5 Selected statistical parameters of pressure measurements at $y / D=0.35$ and differences between the empirical probability distribution function $(E)$ and the corresponding Normal $(N)$ and Gumbel $(G u)$ fits for negative and positive extreme pressures (data set of three times 65,536 points sampled at $1 \mathrm{kHz}, \approx 3.25 \mathrm{~min}$ )

\begin{tabular}{|c|c|c|c|c|c|c|c|c|c|c|}
\hline \multirow[t]{2}{*}{$Y / D$} & \multirow[t]{2}{*}{$V_{0}(\mathrm{~m} / \mathrm{s})$} & \multirow[t]{2}{*}{$C_{\mathrm{s}}$} & \multirow[t]{2}{*}{ K } & \multirow[t]{2}{*}{$z_{\min }^{\prime}(-)$} & \multirow[t]{2}{*}{$z_{\text {max }}^{\prime}(-)$} & \multicolumn{2}{|l|}{$\Delta z^{\prime}{ }_{0.1 \%}$} & \multicolumn{2}{|l|}{$\Delta z_{99.9 \%}^{\prime}$} & \multirow{2}{*}{$\begin{array}{l}\text { Close to Gaussian } \\
\text { pdf }\end{array}$} \\
\hline & & & & & & {$[E-N]$} & {$[E-G u]$} & {$[E-N]$} & {$[E-G u]$} & \\
\hline \multirow[t]{2}{*}{2.8} & 24.6 & -0.48 & 0.45 & -6.36 & 3.72 & -1.34 & -2.79 & -0.50 & -1.30 & $\approx P(0.05-0.90)$ \\
\hline & 29.5 & -1.10 & 1.44 & -5.65 & 3.70 & - & - & - & - & - \\
\hline 5.6 & 29.5 & -1.06 & 2.05 & -7.88 & 4.78 & 1.55 & -2.72 & -0.55 & -2.60 & $\approx P(0.10-0.90)$ \\
\hline 6.9 & 29.5 & -0.88 & 0.39 & -3.70 & 3.01 & -0.06 & -1.27 & -1.11 & -1.11 & $\approx P(0.25-0.75)$ \\
\hline 8.3 & 29.5 & -1.02 & 0.44 & -4.34 & 3.11 & - & - & - & - & - \\
\hline 9.3 & 29.5 & 0.86 & 0.27 & -2.46 & 4.71 & 2.32 & 0.09 & -1.76 & -1.50 & $\approx P(0.25-0.75)$ \\
\hline
\end{tabular}



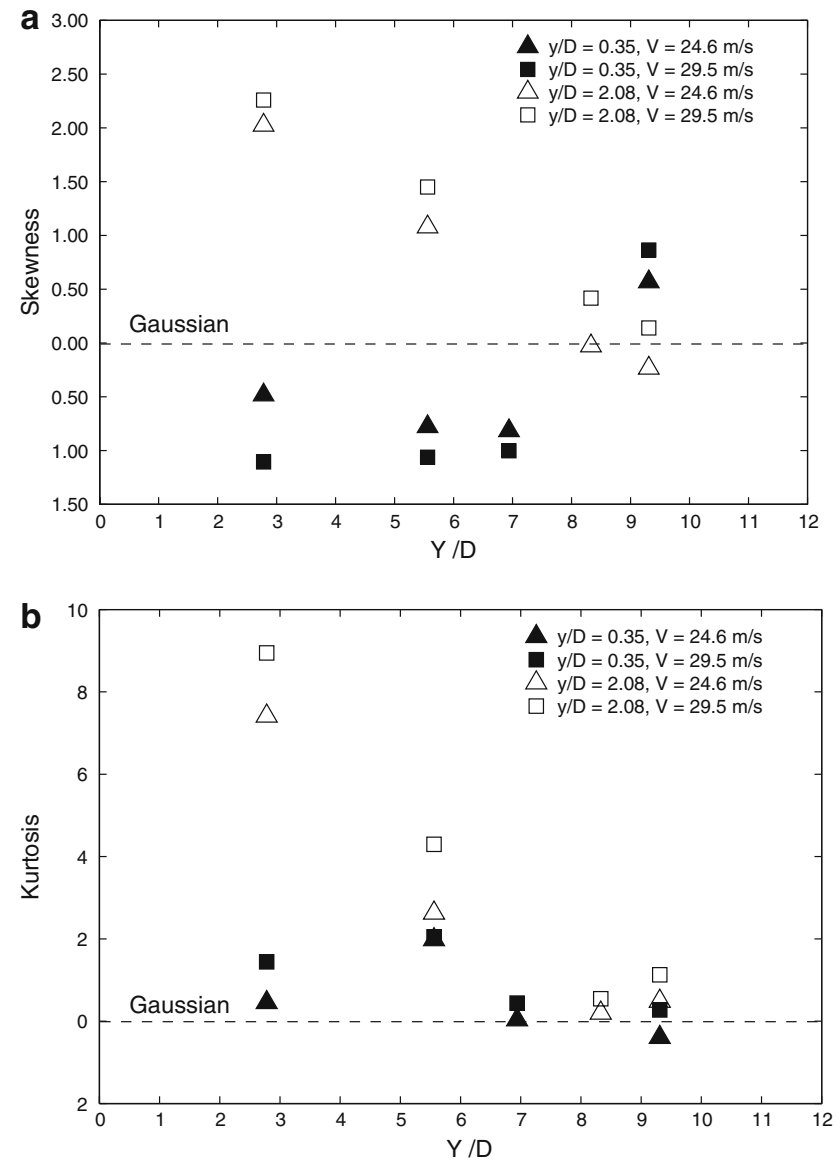

Fig. 6 Evolution of skewness $C_{\mathrm{s}}$ (top) and kurtosis $K$ (bottom) with relative pool depth $Y / D$

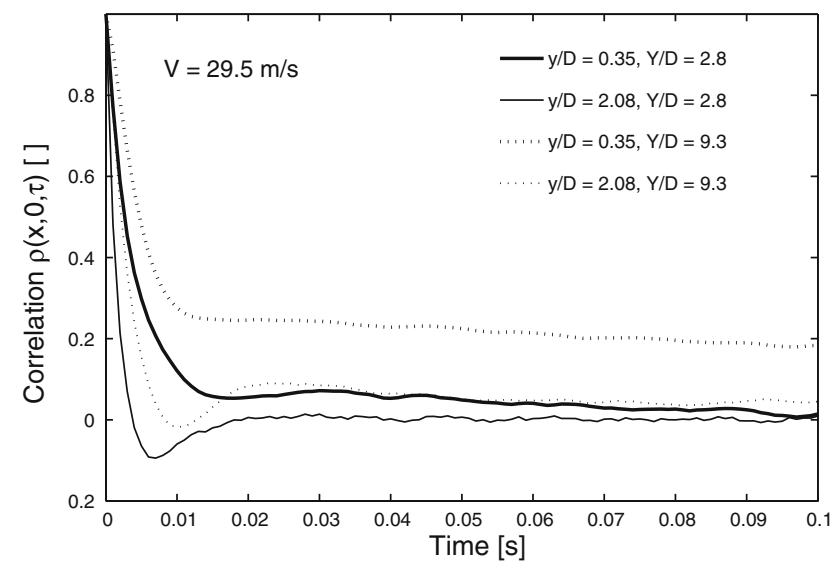

Fig. 7 Normalized time correlation function of dynamic pressures at impact onto a shallow pool $(Y / D=2.8)$ and a deep pool $(Y / D=9.3)$, at $y / D=0.35$ and 2.08 , created by a plunging jet with $V=29.5 \mathrm{~m} / \mathrm{s}$ (from the expected value FFT of three files of 65,536 points each, $f=1 \mathrm{kHz}$ )

the stagnation point (also observed by Melo 2001; Kamoi and Tanaka 1972), due to jet core instability and streamline deflection.
At $y / D=2.08$, the autocorrelation rapidly becomes negative. This corresponds to the impact of the turbulent shear layer created by jet diffusion inside the pool. However, due to the deflection of the plunging jet a wall jet is formed, which lessens the impact of the turbulent shear layer with the bottom. Therefore, only small duration pressure oscillations are measured which are non-correlated. Above the wall jet, a large roller was perceived during the tests. Further away from the jet axis, the wall jet detaches from the bottom.

For a deep pool $(Y / D=9.3)$, pressures at the bottom reflect the impact of a developed turbulent shear layer. Close to the axis $(y / D=0.35)$, skewness is positive and the autocorrelation drops also sharply. Small local fluctuations reflect the low turbulence level at the centre of the developed jet, whereas the mild decay of the autocorrelation may correspond to a long persisting wall jet. At $y / D=2.08$, farther from the jet axis, skewness is almost zero and the autocorrelation becomes negative at about $0.01 \mathrm{~s}$. The transducer is under the bottom wall jet. Compared to the equivalent situation for shallow pools, the autocorrelation crosses zero at about twice the time lag. This means that the overrunning flow structure is slightly more persistent in deep pools than in shallow pools, indicating the presence of slower (i.e. larger) flow structures. Thus, the rotating flow cell above the wall jet is larger for deep pools than for shallow pools. A schematic summary of the two extreme cases of a shallow pool $(Y / D=2.8)$ and a deep pool $(Y / D=9.3)$ is shown in Fig. 8 .

\subsection{Core persistence}

The persistence (core length, $x_{\mathrm{c}}$ ) of a rough turbulent plunging jet is not precisely defined. Because no direct measurements exist, $x_{\mathrm{c}}$ is often assumed to be about 5D. Ervine and Falvey (1987) indicate a core contraction angle of $7-9^{\circ}$ for variable air entrainment conditions based on an $\alpha_{2} / \alpha_{1}$ ratio obtained from momentum considerations, giving an estimate for core length of about 3-4 diameters. In the present case, the core development length is assumed to be $Y / D=5.6$ corresponding to the highest kurtosis of pressure measurements at stagnation (Table 5). In turbulent flows, deviations from a Gaussian pdf in the tails is a sign of intermittency (Carbone et al. 2000), which is directly related to the kurtosis. Intermittent and sporadic extreme negative and positive pressures generated by intermittent flow motion tend to significantly increase the 4th statistical moment. This occurs precisely at the transitional depth for which the core is interrupted by turbulent flow structures. The corresponding pressure measurements time series shows 
Fig. 8 Schematic representation of flow patterns and statistical parameters as $C_{\mathrm{s}}$ and $K$ for two envelope pool depths $Y / D$. Detail of presumed local flow pattern at the stagnation point, including fluctuating pattern due to jet instability

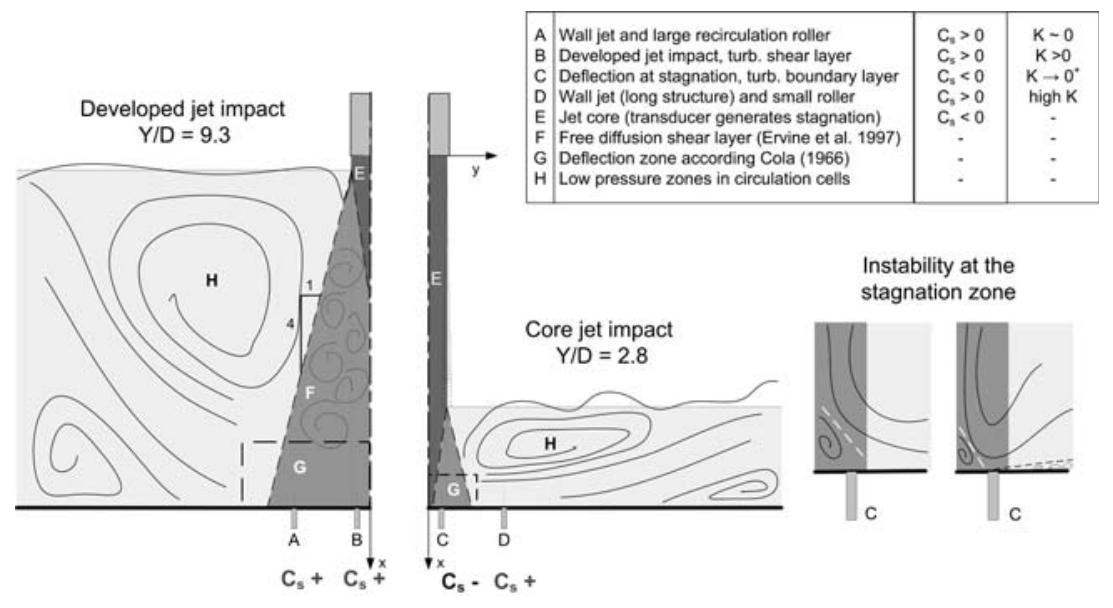

(very) low pressure extreme values compared to the mean (up to $-8 \sigma$ ) and also very high positive extreme values (up to $4.8 \sigma$ ). This core length is larger than the value reported by Ervine and Falvey (1987) for free diffusion conditions. Since jet deflection at the vicinity of the pool bottom occurs in quasi-constant energy conditions, the core length in limited-depth pools should be computed by adding the free diffusion core length and the depth (thickness) of the jet deflection zone. Although this thickness is not well-characterized for turbulent plunging jets, it can be assumed to be about $0.25 Y$ for submerged jets according to Cola (1996) and Gutmark et al. (1978).

\subsection{Radial pressure distribution}

Figure 9 shows that at $y / D=0.35, C_{\mathrm{s}}$ is only positive for deep pools. At $y / D=2.08$, skewness is either positive or close to zero, corresponding to the impact of the shear layer of the jet. The highest $C_{\mathrm{s}}$ at $y / D=2.08$ corresponds to $Y / D=2.8$ (shallow), for which the bottom wall jet is disturbed by pool surface oscillations. Similarly, the kurtosis at $y / D=2.08$ is also higher for shallow pools (more intermittent). For increasing submergence, $C_{\mathrm{s}}$ and $K$ at $y / D=2.08$ tend to zero (i.e. to a Gaussian pdf), reflecting the establishment of a well-developed wall jet. Figure 10 shows the evolution of $C_{\mathrm{s}}$ and $K$ with velocity. At stagnation, $C_{\mathrm{s}}$ presents little scatter for each pool depth investigated. The only case of change of sign of the skewness, and therefore of flow pattern above the transducer, corresponds to $Y / D=8.3$ at $y / D=0.35$. In these flow conditions, strong recirculation inside the basin lowered the pool surface of 10 to $20 \mathrm{~cm}$ at the point of jet entry, reducing the diffusion length locally. Therefore, jet impact became similar to that in shallower pools. This effect was only relevant at this relative pool depth and is considered an artefact of the facility. At $y / D=2.08, C_{\mathrm{s}}$ considerably increases with velocity in shallow pools, most likely due to the increasing pool surface instability. In terms of kurtosis, intermittency is higher at $y / D=0.35$ for the intermediate pool of $Y / D=5.6$ and for almost all
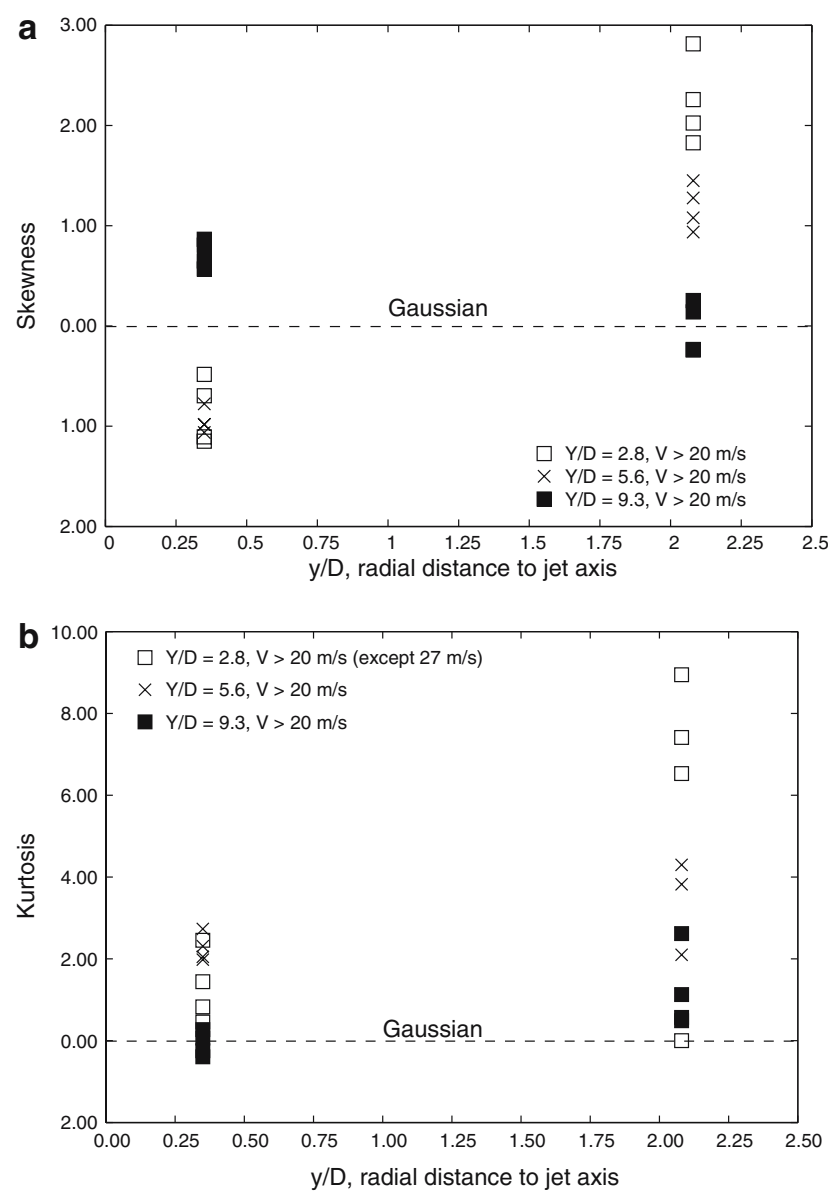

Fig. 9 Evolution of skewness $C_{\mathrm{s}}$ (top) and kurtosis $K$ (bottom) with radial distance from jet axis 

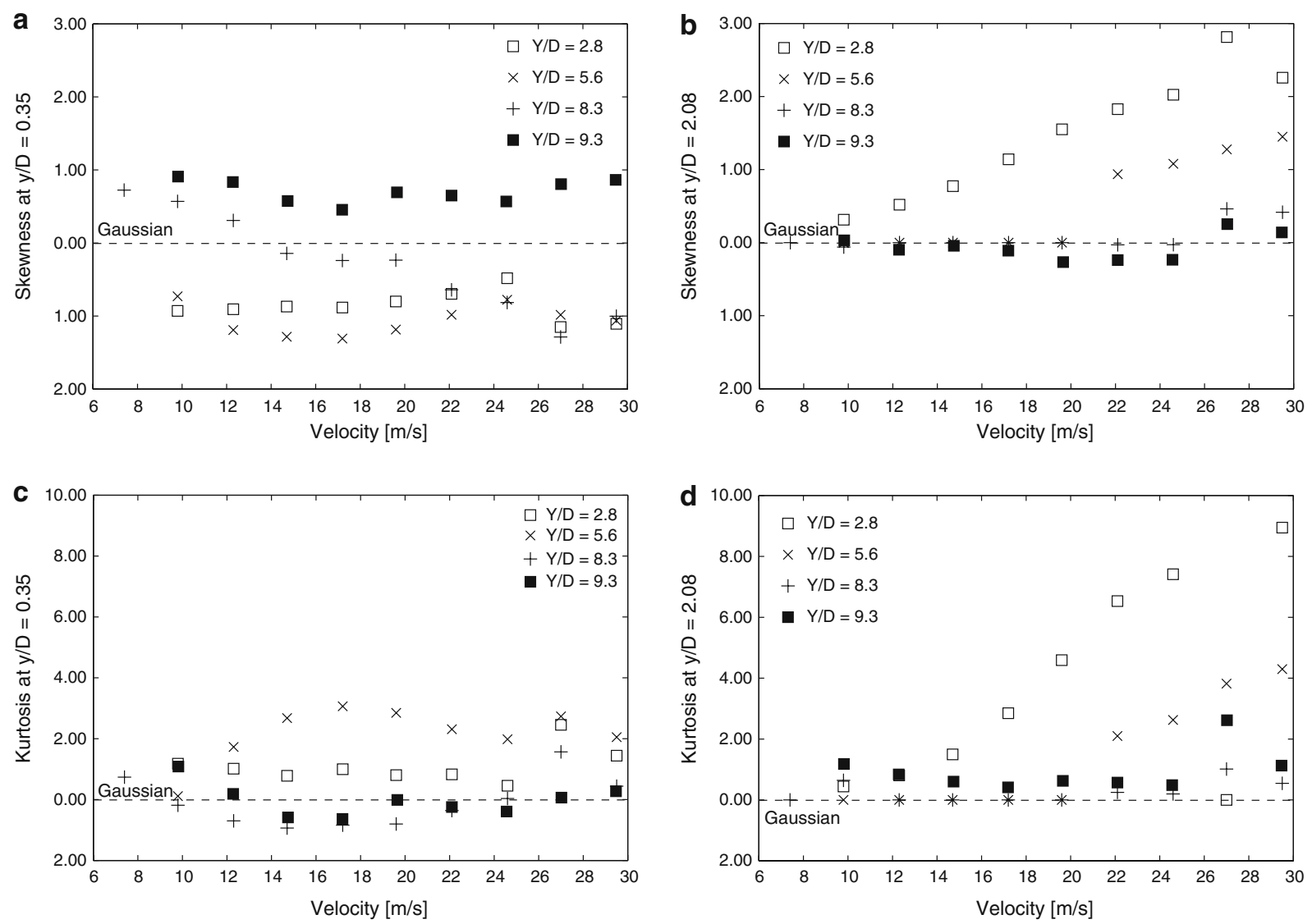

Fig. 10 Evolution of skewness $C_{\mathrm{s}}$ and kurtosis $K$ with velocity (or $F r$ as indicated in Table 1 ) as function of pool depth: left, $y / D=0.35$; right, $y / D=2.08$

velocities tested. For the remaining $Y / D$ values, the kurtosis are close to zero, corresponding either to core jet impact or to developed turbulence for respectively shallow and deep pools. At $y / D=2.08, K$ increases considerably with velocity for shallow pools. Bottom wall jets are reflected by the facility's side walls and generate significant pool surface oscillations and air entrainment. For deep pools, the kurtosis (and thus the intermittency of the flow) is significantly reduced due to submergence and lower air content at the pool bottom.

The previous interpretations indicate that wall pressure skewness is positive for predominantly incident flow and negative when the flow is predominantly moving away from the pool bottom, as previously observed by Lopardo and Henning (1985) and Fiorotto and Rinaldo (1992) under hydraulic jump rollers.

\section{Conclusions}

Plunging jets were experimentally investigated using velocities and aeration conditions similar to those in prototype water releasing structures of dams at various pool depths. Dynamic pressure measurements at issuance and at the bottom of the pool allow concluding the following:

1. Dynamic pressures both at the nozzle exit and at the bottom in shallow and deep pools follow a Gaussian (Normal) distribution in the intermediate range of cumulated probability $(|z| \leq 1.5)$.

2. Impact pressures under the jet have negative skewness in shallow pools and positive skewness in deep pools.

3. The kurtosis under the jet axis reflects the intermittent character of the flow; for increasing submergence, the maximum kurtosis provides an estimate of the core development length in limiteddepth plunge pools with flat bottom.

4. The double-structure time autocorrelation function of pressure measurements in shallow pools and the negative sign of the skewness suggests an acute deflection of the jet streamlines and the formation of a turbulent boundary turbulent layer at the bottom. 
The interpretation of wall pressure skewness values allowed inferring the pool bottom zones under downward and upward moving currents. This interpretation has been used to investigate the flow patterns in pools with more complex geometry in highly aerated turbulent flows (Manso 2006). Performing tests at nearprototype conditions in terms of velocity and aeration conditions allows obtaining reliable impact pressure data. However, it increases the practical difficulties of performing air-water flow measurements. Further understanding of the effects of air entrainment in wall pressure distributions requires the analysis of local airwater measurements obtained with the same flow conditions.

Acknowledgments The first author acknowledges the Portuguese Foundation for Science and Technology (FCT) for the fellowship 21847/2005. The experimental work performed at the LCH-EPFL was co-funded by FCT grant 6894/2001 and the Swiss Federal Office of Energy. The suggestions by Prof. Virgilio Fiorotto from the University of Trieste (Italy) and by the anonymous reviewers are acknowledged.

\section{References}

Albertson ML, Dai YB, Jensen RA, Rouse H (1948) Diffusion of submerged jets. Trans ASCE 115:639-697

Arndt REA, Ippen AT (1970) Turbulence measurements in liquids using an improved total pressure probe. J Hydraul Res 8(2):131-158

Bollaert EFR, Schleiss AJ (2003) Scour of rock due to the impact of plunging high velocity jets, Part 2: experimental results of dynamic pressures at pool bottoms and in one- and twodimensional closed end rock joints. J Hydraul Res 41(5):465-480

Carbone V, Regnoli G, Martines E, Antoni V (2000) Intermittency and self-similarity in plasma edge fluctuations. Phys Plasmas 7(2):445-447. DOI S1070-664X(00)04401-3

Carreras BA, Milligen BP, Pedrosa MA, Balbín R, Hidalgo C, Newman DE, Sanchéz E, Frances M, García-Cortés I, Bleuel J, Endler M, Riccardi C, Davies S, Matthews GF, Martines E, Antoni V, Latten A and Klinger T (1998) Selfsimilarity of the plasma edge fluctuations. Phys Plasmas 5(10):3632-3643. DOI S1070 -664X(98)00510-2

Chanson H, Aoki S, Hoque A (2004) Physical modelling and similitude of air bubble entrainment at vertical circular plunging jets. Chem Eng Sci 59:747-758
Cola R (1966) Diffusion of a vertical plane jet in a water pool of limited depth (in Italian). L'Energia Elettrica 43(11):649_ 664

Ervine A, Falvey HT (1987) Behaviour of turbulent water jets in the atmosphere and in plunge pools. Proc Inst Civil Eng Pt 2 83:295-314

Ervine DA, Falvey HT, Withers W (1997) Pressure fluctuations on plunge pool floors. J Hydraul Res 35(2):257-279

Fiorotto V, Rinaldo A (1992) Turbulent pressure fluctuations below hydraulic jumps. J Hydraul Res 30(4):499-520

Gutmark GE, Wolfshtein M and Wygnanski I (1978) The plane turbulent impinging jet. J Fluid Mech 88(4):737-756

Hartung F, Häusler E (1973) Scours, stilling basins and downstream protection under free overfall jets at dams. In: ICOLD (ed) Proceedings of the 11th congress on large dams, Madrid, Q41. vol R3, pp 39-56

Kamoi A, Tanaka H (1972) Measurements of wall shear stress, wall pressure and fluctuations in the stagnation region produced by oblique jet impingement. In: Cockrell DJ (ed) Fluid dynamic measurements conference papers, vol 1, pp 217-227

Lopardo RA, Henning RE (1985) Experimental advances on pressure fluctuations beneath hydraulic jumps. In: Proceedings of the 21st IAHR Congress, Melbourne, vol 3, pp 633638

Manso PA (2006) The influence of pool geometry and induced flow patterns on rock scour by high-velocity plunging jets. Ph.D. thesis no. 3430, Ecole Polytéchnique Fédérale de Lausanne (EPFL)

May RWP, Willoughby IR (1991) Impact pressures in plunge basins due to vertical falling jets. Report SR242, HR Wallingford

McKeogh EJ, Elsawy EM (1980) Air retained in pool by plunging water jet. J Hydraul Div 106(10):1577-1593

McKeogh EJ, Ervine DA (1981) Air entrainment rate and diffusion pattern of plunging liquid jets. Chem Eng Sci 36:1161-1172

Melo JF (2001) Hydrodynamic loads acting on floor slabs of energy dissipation basins by plunging jets (in Portuguese). $\mathrm{Ph}$.D. thesis, Instituto Superior Técnico (IST), Lisbon

Melo JF (2002) Reduction of plunge pool floor dynamic pressure due to jet air entrainment. In: Bollaert EFR, Schleiss AJ (eds) Rock scour due to falling high-velocity jets, Swets and Zeitlinger, Lisse, pp 125-136

Puertas-Agudo J (1994) Hydraulic criteria for the design of energy dissipation basins in arch dams with free crest overfall (in Castilian). Ph.D. thesis, Universitat Politécnica de Catalunya, Barcelona

Toso JW, Bowers CE (1988) Extreme pressures in hydraulicjump stilling basins. J Hydraul Eng 114(8):829-843 